\title{
SATISFACCIÓN DE LOS ALUMNOS DE ENFERMERÍA DE LA UNIVERSIDAD CATÓLICA SAN ANTONIO (UCAM) SOBRE SU FORMACIÓN PRÁCTICA. SEMINARIOS EN SALAS DE DEMOSTRACIONES Y PRÁCTICAS CLÍNICAS
}

PRACTICAL TRAINING SATISFACTION OF NURSING STUDENTS AT THE CATHOLIC UNIVERSITY OF SAN ANTONIO (UCAM) SEMINARS IN DEMOSTRATION CLASSES AND CLINICAL TRAINING.

*Lorente Gallego, AM., " ${ }^{* *}$ García Sánchez, C., **López Arroyo, MJ., ****Echevarria

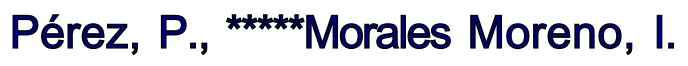

*Lcda. en Documentación. Profesora Colaboradora. **Enfermera. Lcda. en Antropología. Profesora Colaboradora. $* *$ Enfermera. Lcda. en Antropología. Subdirectora y Coordinadora. $* * * *$ Enfermera. Doctora en Antropología. Directora. $* * * *$ Enfermera. Lcda. en Antropología. Secretaria. Titulación de Enfermería. Universidad Católica San Antonio. Murcia.

Palabras clave: Estudiantes de Enfermería, Satisfacción, Prácticas clínicas, Universidad Católica San Antonio.

Keywords: Nursing Students, Satisfaction Clinical training, and Catholic University of San Antonio.

\section{RESUMEN}

Objetivo. Conocer la satisfacción de los alumnos de enfermería de la Universidad Católica San Antonio sobre las prácticas que desarrollan durante su formación de pregrado. Método. Se realizó un estudio descriptivo observacional trasversal realizado en la Diplomatura de Enfermería de la Universidad Católica San Antonio durante los cursos académicos 2004/05 y 2007/08. La población objeto de estudio son todos los alumnos de enfermería matriculados en asignaturas de segundo y tercer curso con créditos prácticos. Para la realización del estudio se elaboró un cuestionario específico cerrado de respuesta múltiple. Resultados. Más del $90 \%$ de los alumnos afirman que los seminarios mejoran el inicio de las prácticas clínicas, y que el estudio teórico de los seminarios prácticos es de gran ayuda para mejorar la técnica. Las técnicas aprendidas en los seminarios se corresponden con la realidad asistencial. Los alumnos de ambos cursos académicos califican la relación con los profesionales de los centros sanitarios y socio-sanitarios como buena y muy buena en más de un $90 \%$ de los casos. Más del $80 \%$ de los alumnos consideran que el profesional - tutor ha facilitado su integración en el equipo de trabajo. Conclusiones. Se puede subrayar que la satisfacción de los alumnos tanto en las prácticas internas y externas es buena y que la relación con los profesionales - tutores es adecuada. En cuanto a la opinión de los alumnos sobre las 
gestiones que se llevan a cabo desde la Unidad de Prácticas de Enfermería, concluimos que existen aspectos mejorables sobre los que ya hemos comenzado a trabajar.

\section{ABSTRACT}

Objective: To know the satisfaction of UCAM nursing students regarding the clinical training included in the practical training they attended during the undergraduate program. Method: A descriptive, observational and transversal study was carried out with nursing students of the Catholic University of San Antonio during the academic years 2004/05 and 2007/08. The studied sample consisted of all the nursing students registered in courses of second and third year with clinical training credits. A closed specific questionnaire of multiple choice was elaborated for the accomplishment of the study. Results: More than $90 \%$ of the students state that seminars improve the clinical training and that the theoretical study of practice seminars is helpful for improving nursing techniques. Techniques learned at seminars meet real assistance. Students of both academic years describe the relation with nurses and clinical mentors at hospitals and health centers as good and very good in more than $90 \%$ of the cases. More than $80 \%$ of the students consider that clinical mentors have facilitated its integration in working team. Conclusions: It is important that the student satisfaction in internal and external practice training is good and that the relation with clinical mentors is adequate. Regarding the opinion of the students about the clinical mentor management in the Unit of Nursing Practical Training, it was concluded that there are aspects to be improved in which we have already begun to work.

\section{INTRODUCCIÓN}

La realización de prácticas clínicas supone un hecho de vital importancia en el curriculum de los estudiantes de Ciencias de la Salud, brindando a los alumnos la oportunidad de realizar técnicas, adquirir conocimiento, desarrollar habilidades y aprender actitudes, dentro de un complejo entorno sanitario y socio-sanitario que desconocen y que en ocasiones no resulta todo lo receptivo como ellos esperan.

La preparación y formación previa a la salida del alumno al mundo real supone uno de los grandes retos de la docencia, pero el objetivo de este reto no puede quedar únicamente para el alumno egresado, sino que es necesario que el alumno de pregrado complete su formación teórico-práctica en entornos reales.

La realización de seminarios prácticos en salas de demostraciones representa para el alumno una primera toma de contacto con los materiales y técnicas que posteriormente desarrollará, en un ambiente controlado, donde el alumno puede realizar la técnica cuantas veces sea necesario sin que esto suponga un riesgo o una molestia para el paciente.

La utilización de estas situaciones simuladas permite mejorar la calidad del proceso de aprendizaje. La pirámide de aprendizaje de Bales(1) establece que la tasa de retención para los ejercicios prácticos supone un $75 \%$, siendo uno de los porcentajes más altos con respecto a otras formas de aprendizaje que se podrían realizar en sala de prácticas como es la demostración de docente de la técnica sin repetición posterior del alumno que solo supone un $50 \%$ de la tasa de retención. Esta situación nos obliga a tratar los seminarios prácticos no como situaciones aisladas del proyecto docente, sino que deben tener una planificación lógica y coherente no sólo con el plan de estudios sino con la asignatura a la que se correspondan dichas prácticas. 


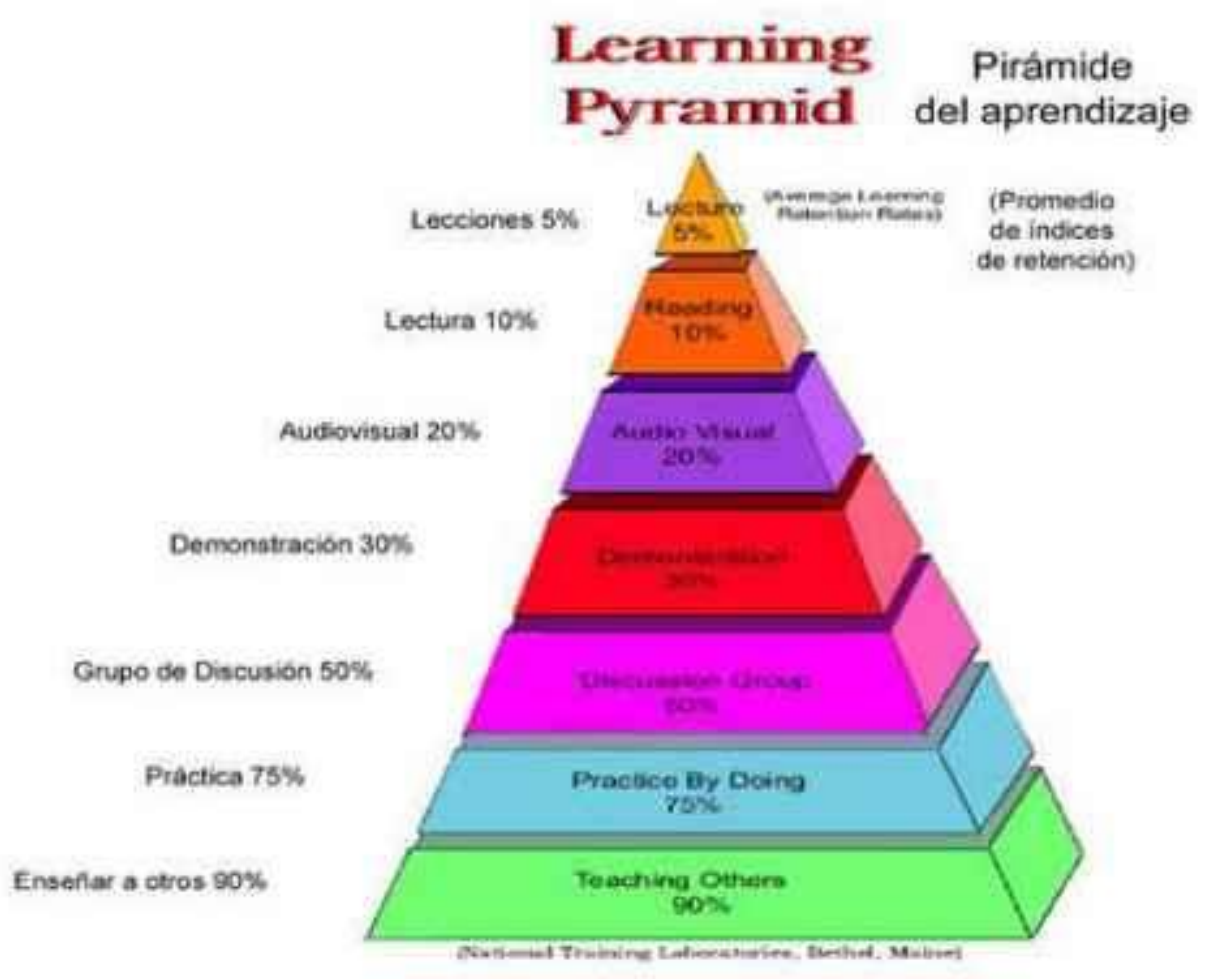

Figura 1. Pirámide de Aprendizaje.

Los estudiantes de enfermería de la UCAM realizan un total de 226,5 créditos durante los tres años de la Diplomatura, de estos un $31 \%$ corresponde a créditos prácticos, que dividimos en prácticas internas o seminarios prácticos en sala de demostraciones con simuladores y prácticas clínicas externas o prácticas en centros sanitarios y sociosanitarios de la Región de Murcia.

La gestión de todos los créditos prácticos se realiza a través de la Unidad de Prácticas de Enfermería (UPE), compuesta por personal administrativo y docente coordinado por la subdirectora de la Diplomatura. Esta unidad tiene entre sus funciones la realización de cronogramas de prácticas de alumnos tanto internas como externas, difusión de dicha información a profesores responsables de asignaturas, alumnos y centros sanitarios y socio-sanitarios donde se realizan prácticas y la atención al alumno en todas aquellas cuestiones relacionadas con prácticas.

Durante el primer año, los seminarios prácticos se concentran principalmente en la asignatura de Fundamentos de Enfermería. El alumno comienza los seminarios prácticos desde el principio del curso y una vez finalizados realizará un primer examen de corte teórico, sobre los contenidos explicados con anterioridad. Superado este examen el alumno debe someterse a un examen práctico, donde debe demostrar que ejecuta las técnicas correctamente y es capaz de resolver las dudas o problemas que pueden surgir durante la realización del mismo.

Este examen capacita al alumno para la realización de prácticas clínicas externas, no sólo para la asignatura de Fundamentos de Enfermería sino también para todas las asignaturas de los cursos siguientes, siendo las asignaturas de Enfermería Clínica del Adulto I y II en 
segundo y tercero respectivamente las que concentran el mayor número de créditos prácticos tanto en prácticas internas o seminarios como prácticas externas.

En ambas asignaturas el alumno debe superar un examen práctico como parte de la evaluación de dichas asignaturas.

Esta forma de evaluación se basa en el modelo de competencia de Georges Miller ${ }^{(2)}$, que parte de una estructura piramidal donde los dos primeros grupos forman la base correspondiente al conocimiento (saber y saber como) para pasar a un tercer escalón, donde el alumno pueda demostrar que no sólo posee los conocimientos necesarios, sino también las actitudes y habilidades necesarias para el desarrollo de la técnica (demuestra como), lo que le capacitará y ayudará al desarrollo posterior en un ámbito real (hace).

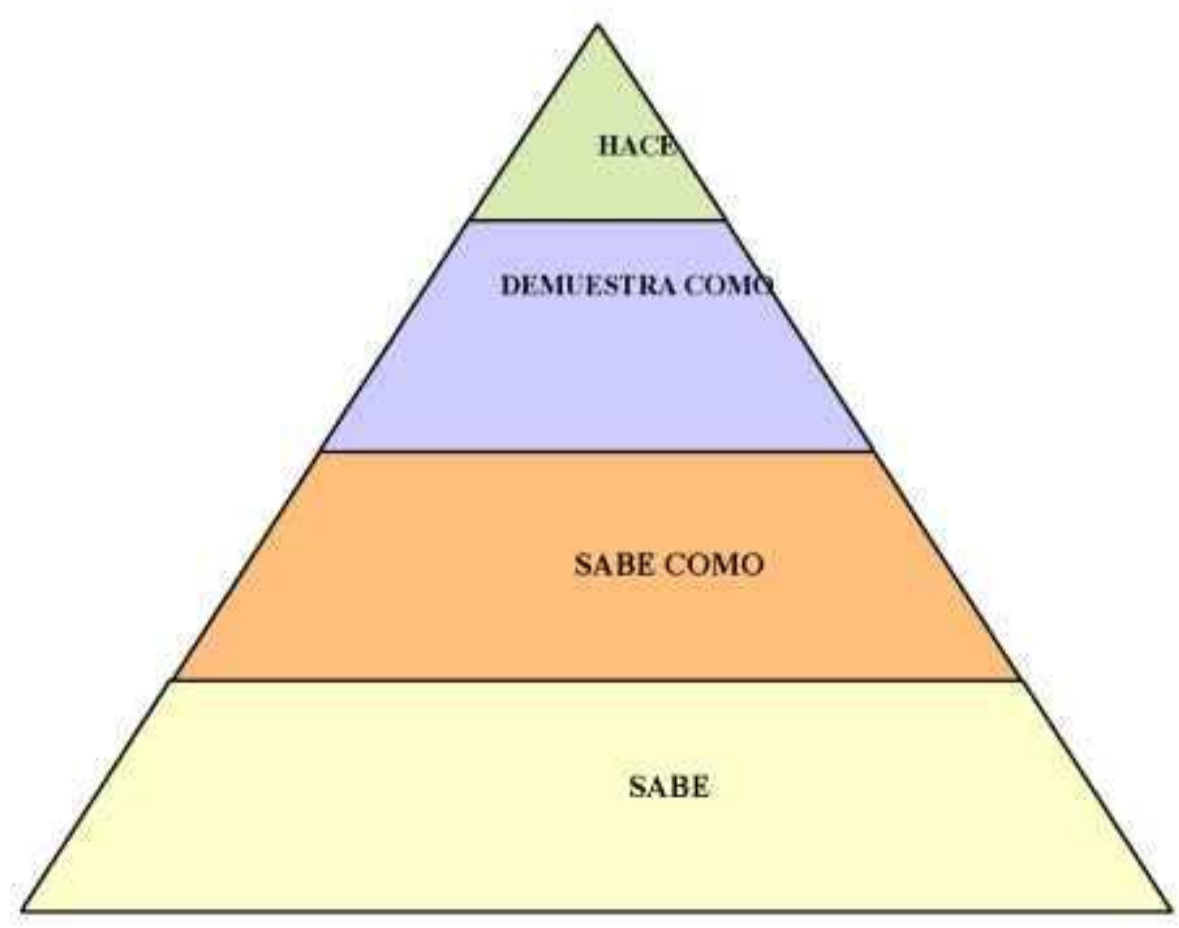

Piranide de Villes G. 1990

\section{Figura 2. Pirámide de Millar G.}

El objetivo general de este estudio es conocer la satisfacción de los alumnos de enfermería de la UCAM sobre las prácticas que desarrollan durante su formación de pregrado.

Como objetivos específicos:

- Conocer la satisfacción de los alumnos en las prácticas internas o seminarios prácticos en sala de demostraciones.

- Conocer la satisfacción de los alumnos en las prácticas clínicas que realizan en centros sanitarios y socio-sanitarios de la Región de Murcia, durante su formación. 
- Conocer la opinión de los alumnos con respecto a la gestión de las prácticas internas y externas por parte de la UPE.

- Conocer la evolución de la satisfacción de los alumnos respecto a su formación práctica.

\section{MATERIAL Y MÉTODO}

Se realizó un estudio descriptivo observacional trasversal realizado en la Diplomatura de Enfermería de la UCAM durante los cursos académicos 2004/05 y 2007/08.

En el curso académico 2004/05 la Diplomatura de Enfermería, con la intención de mejorar la formación práctica y conocer la opinión de los alumnos respecto a la misma, realizó una encuesta de la que se derivaron una serie de cambios, que volvieron a ser analizados tres cursos académicos después.

La población objeto de estudio se compone de todos los alumnos de enfermería matriculados en asignaturas de segundo y tercer curso con créditos prácticos internos y externos. Un total de 342 alumnos en el curso académico 2004/05 y 432 alumnos en el curso académico $2007 / 08$.

Para la realización del estudio se elaboró un cuestionario específico cerrado de respuesta múltiple.

La cumplimentación por parte de los alumnos de dicho cuestionario se realizó en el mes de Mayo de cada curso académico, con la colaboración de los profesores responsables de asignaturas troncales de segundo y tercer curso.

En el curso académico 2004/05 el cuestionario se realizó a 194 (56.7\%) alumnos, de los que 88 (44\%) pertenecen a segundo y 106 (55\%) a tercero. En el curso académico 2007/08 el cuestionario se realizó a un total de 245 (56.7\%) de los alumnos, de los que 98 (40\%) pertenecen a segundo y $147(60 \%)$ de tercero.

El análisis de los datos se realizó a través del programa estadístico SPSS versión 13.

\section{RESULTADOS}

La opinión de los alumnos de segundo y tercero de enfermería sobre los seminarios prácticos que se realizaron en la Universidad durante el curso académico 2004/05 fue de muy satisfactoria en un $10 \%$ y un $16 \%$ respectivamente, y nada satisfactorios en un $13 \%$ y un $6 \%$. En el curso académico 2007/08 el 22\% de los alumnos de ambos cursos opina satisfactoriamente sobre los seminarios y un $5 \%$ de estos manifiesta que no son nada satisfactorios.

Tanto en segundo como en tercero de enfermería de los cursos académicos analizados, consideran que los recursos materiales utilizados en los seminarios son suficientes en un $95 \%$. Respecto a su manipulación, en el curso académico 2004/05 ambos cursos afirman en un $60 \%$ que lo manipulan siempre o habitualmente, en el curso académico 2007/08 el $82 \%$ y $66 \%$ de los alumnos de segundo y tercero respectivamente afirman que manipulan el material siempre o habitualmente.

En las gráficas 1 y 2 se representa la opinión de los alumnos de segundo y tercer curso sobre la metodología utilizada al impartir los seminarios. 
Gráfica 1. ¿La metodología utilizada para impartir estos seminarios le parece?. ( $2^{\circ}$ enfermeria)

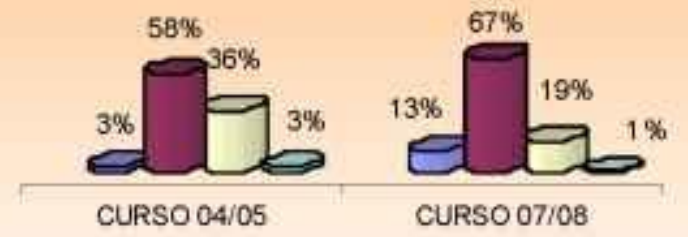

口MUY BUENA $\quad$ BUENA $\square$ REGULAR $\square$ MALA

\section{Gráfica 1. ¿La metodologia utilizada para impartir estos seminarios le parece?. (2" enfermería)}

Gráfica 2. ¿La metodologia utilizada para impartir

estos seminarios le parece? $\left(3^{\circ}\right.$ enfermeria)

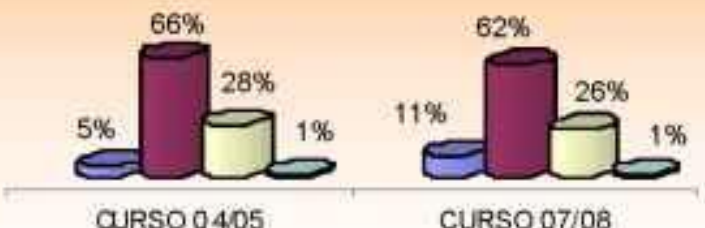

CURSO 0405
CURSO 07/08

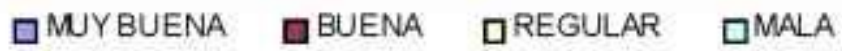

\section{Gráfica 2. ¿La metodología utilizada para impartir estos seminarios le parece? ( $3^{\circ}$ enfermeria)}

Más del $90 \%$ de los alumnos tanto en el curso académico 2004/05 como en el 2007/08, afirman que los seminarios mejoran el inicio de las prácticas clínicas, y que el estudio teórico de los seminarios prácticos es de gran ayuda para mejorar la técnica, gráfica 3 y 4 .

\section{Gráfica 3. Estudiar la teoria de los seminarios prácticos ayuda bastante a la ejecución de la \\ técnica. \\ ( $2^{\circ}$ enfermeria)}

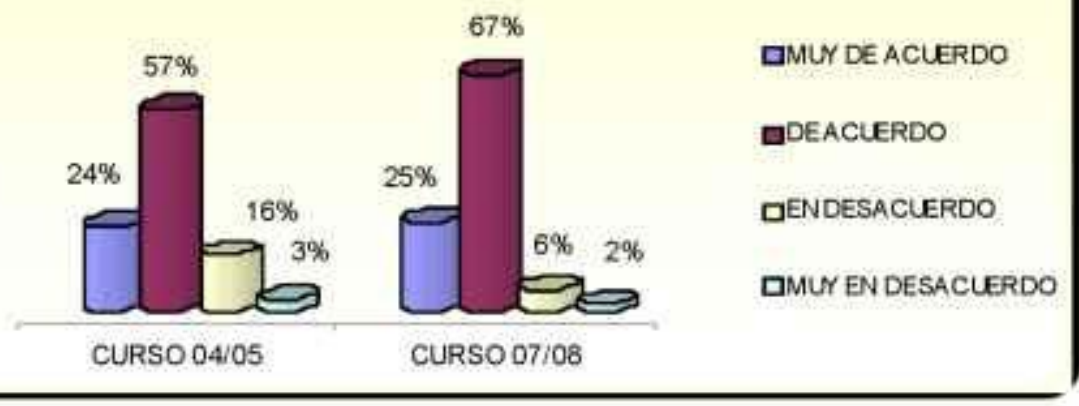

Gráfica 3. Estudiar la teoría de los seminarios prácticos ayuda bastante a la ejecución de la técnica. ( $2^{\circ}$ enfermeria) 


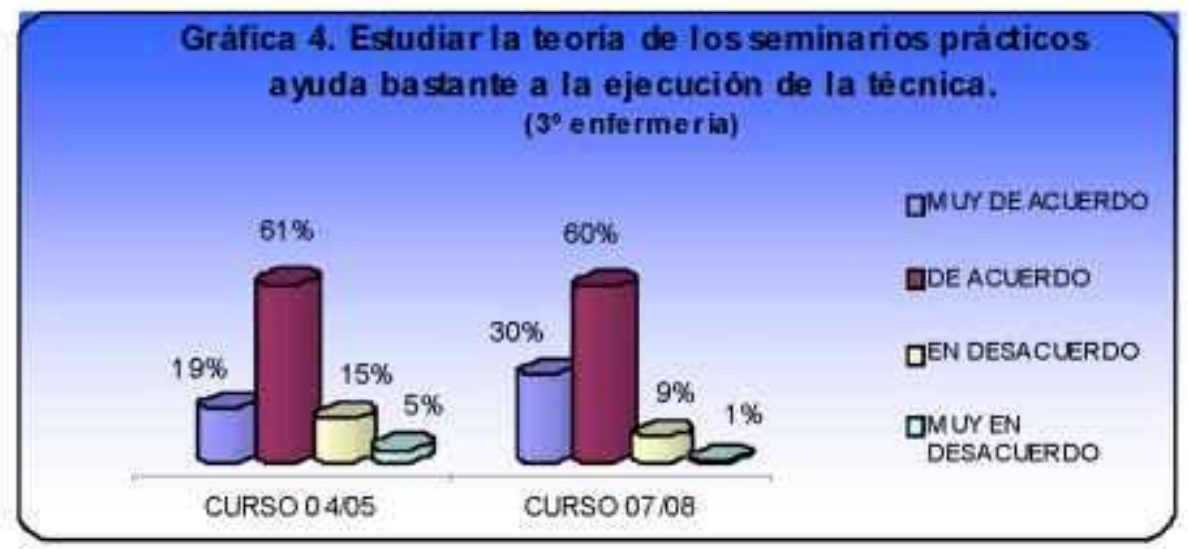

\section{Gráfica 4. Estudiar la teoría de los seminarios prácticos ayuda bastante a la ejecución de la técnica. ( $3^{\circ}$ enfermería)}

Respecto a lo que opinan los estudiantes sobre si las técnicas aprendidas en los seminarios se corresponden con la realidad asistencial, el $44 \%$ de los alumnos de segundo de enfermería, en el curso académicos 2004/05 y el 73\% en el 2007/08 están de acuerdo con esta afirmación, coincidiendo con esta respuesta el $60 \%$ de los alumnos de tercero de enfermería en ambos cursos académicos.

Respecto a la afirmación "Los canales de comunicación de los alumnos con la universidad en el periodo de prácticas extrauniversitarias están definidos y son fluidos", en el curso académico $2004 / 05$ el $32 \%$ de los alumnos de segundo y el $52 \%$ de los de tercero estaban de acuerdo con la afirmación, un 55\% de alumnos del curso académico 2007/08 para ambos cursos tienen la misma opinión.

Más del $50 \%$ de los alumnos de segundo y tercero de enfermería, en ambos cursos académicos, afirman contar con los documentos teóricos sobre los que se desarrollan las prácticas con anterioridad a la misma. El $70 \%$ de los alumnos encuestados afirman no haber estudiado nunca o casi nunca la documentación teórica aun teniéndola con anterioridad.

Más del $90 \%$ de los alumnos encuestados en ambos cursos académicos estudiados, manifiestan que el grado de satisfacción respecto a sus prácticas externas es Muy bueno y bueno.

En la formación de los alumnos de enfermería han participado entre uno y tres tutores de prácticas clínicas en más de un $60 \%$ de los casos. Considerando más del $75 \%$ de los alumnos, que la colaboración de más de un profesional es buena o muy buena.

Los alumnos de ambos cursos académicos califican la relación con los profesionales de los centros sanitarios y socio-sanitarios como buena y muy buena en más de un $90 \%$ de los casos. Más del $80 \%$ de los alumnos consideran que el profesional - tutor ha facilitado su integración en el equipo de trabajo.

En la grafica 5 y 6 se puede observar la opinión de los alumnos sobre la capacitación del profesional / tutor tanto para impartir enseñanzas prácticas como para evaluarlas. 


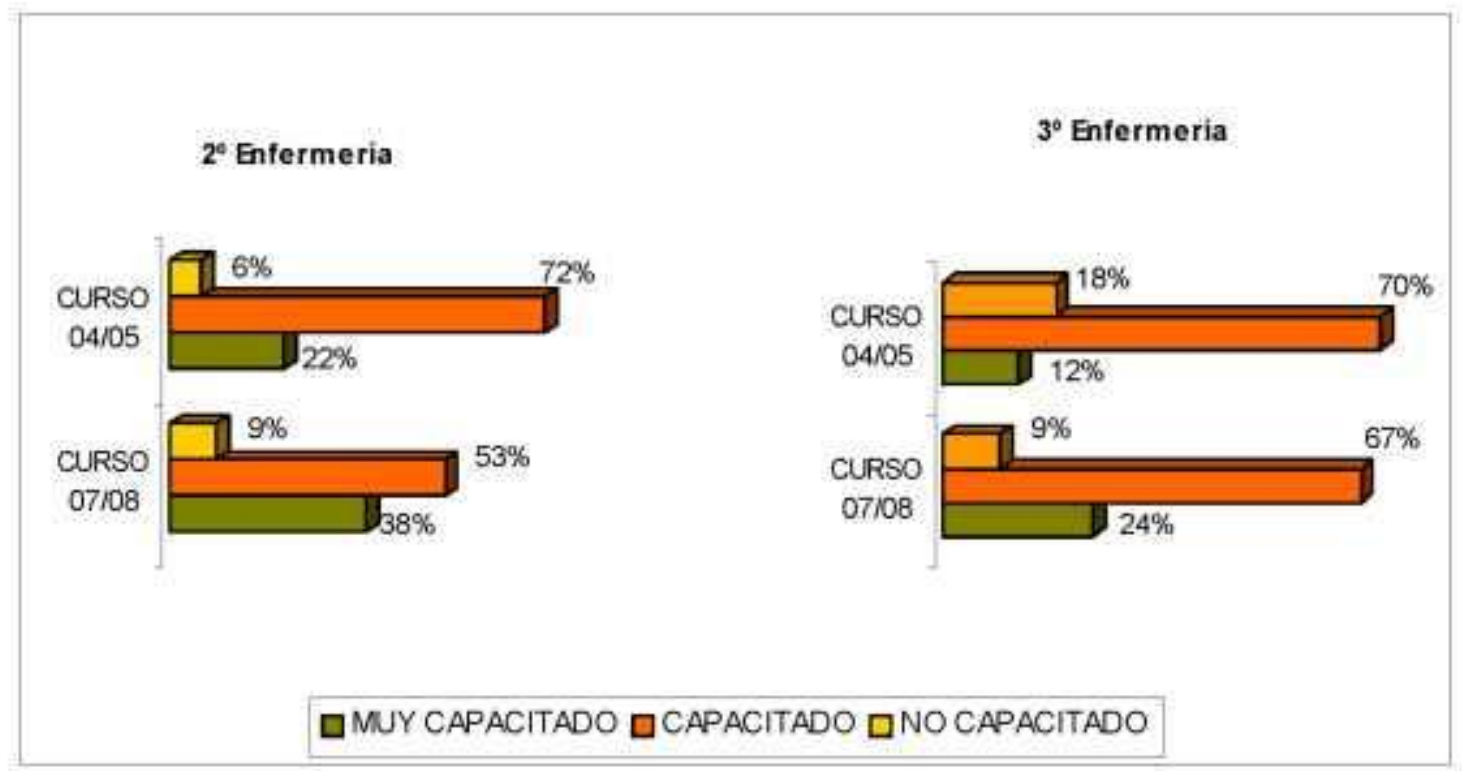

Grafica 5. Considero que el profesor tutor está capacitado para impartir enseñanza

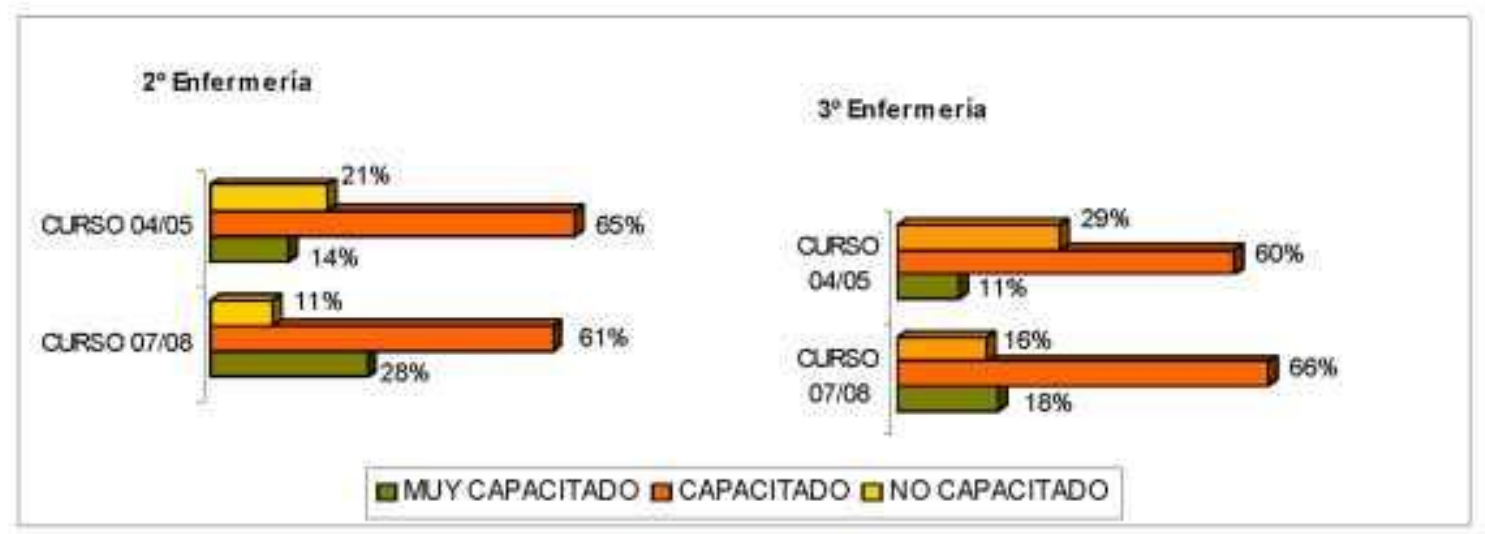

\section{Gráfica 6. Considero que el profesor tutor está capacitado para evaluar las prácticas}

En el curso académico 2004/05 los alumnos de segundo afirmaron estar de acuerdo en un $68 \%$ con el control de firmas y en un $85 \%$ con los criterios de evaluación que cumplimenta el profesional - tutor, considerando la evaluación numérica adecuada en un $85 \%$. Si embargo, los alumnos de tercero ante la misma pregunta afirmaron estar de acuerdo en $62 \%$ y un $65 \%$, considerando la evaluación numérica buena y muy buena en un $66 \%$.

Respecto al curso académico 2007/08 los alumnos de segundo afirmaron estar de acuerdo en un $64 \%$ con el control de firmas y en un $78 \%$ con los criterios de evaluación que cumplimenta el profesional - tutor, considerando la evaluación numérica adecuada en un $75 \%$. Si embargo, los alumnos de tercero ante la misma pregunta afirmaron estar de acuerdo en $65 \%$ y un $77 \%$, considerando la evaluación numérica buena y muy buena en un $68 \%$.

Los ítems evaluados para conocer la satisfacción de los alumnos con respecto al funcionamiento de la UPE, nos aportan los siguientes resultados:

- En el curso académico 2004/05, el $60 \%$ de los alumnos, tanto de segundo como de tercero, considera que los sistemas de difusión de información de los periodos de prácticas 
clínicas y seminarios prácticos le parecen adecuados, considerando el $65 \%$ de los mismos que la información recibida sobre la normativa a seguir para la realización de las prácticas es suficiente. El $60 \%$ de los alumnos consideran insuficiente el horario de atención establecido en la UPE, con respecto al trato recibido, el $40 \%$ de los alumnos piensan que es mejorable.

- En el curso académico $2007 / 08$ el $70 \%$ de los alumnos, tanto de segundo como de tercero, consideran que los sistemas de difusión de información de los periodos de prácticas clínicas y seminarios prácticos le parecen adecuados, de igual modo que un $80 \%$ consideran suficiente la información recibida sobre la normativa a seguir para la realización de las prácticas. El $55 \%$ de los alumnos consideran insuficiente el horario de atención establecido en la UPE, con respecto al trato recibido, el $45 \%$ de los alumnos piensan que es mejorable.

- En cuanto al tiempo de respuestas a las dudas o peticiones realizadas en la UPE, el 55\% de los alumnos encuestados en ambos cursos académicos lo consideran adecuado.

Todos estos datos se resumen en el siguiente cuadro.

\begin{tabular}{|c|c|c|c|c|c|c|c|c|}
\hline \multirow[b]{4}{*}{$\begin{array}{c}\text { Sistemas de } \\
\text { difusión de } \\
\text { información de } \\
\text { prácticas }\end{array}$} & \multicolumn{4}{|c|}{ CURSO ACADÉMICO 2004-05 } & \multicolumn{4}{|c|}{ CURSO ACADÉMICO 2007/08 } \\
\hline & \multicolumn{2}{|c|}{ SEGUNDO } & \multicolumn{2}{|c|}{ TERCERO } & \multicolumn{2}{|c|}{ SEGUNDO } & \multicolumn{2}{|c|}{ TERCERO } \\
\hline & ADECUADOS & INADECUADOS & ADECUADOS & INADECUADOS & ADECUADOS & INADECUADOS & ADECUADOS & INADECT: \\
\hline & $63^{\circ} \mathrm{O}$ & $36 \%$ & $61 \%$ & $32 \%$ & $68^{\circ} \circ$ & $28 \%$ & $73^{\circ} \mathrm{n}$ & $24^{\circ} \mathrm{0}$ \\
\hline \multirow{2}{*}{$\begin{array}{c}\text { Normativa a } \\
\text { seguir para la } \\
\text { realización de } \\
\text { prácticas }\end{array}$} & STFICTENTE & INSIFICIENTE & SUFICIENTK & INSUFICIENTE & SUFICIFNTE & IVSIFICIFNTE & SEFICIENTE & INSIFICII \\
\hline & $62 \%$ & $25^{\circ} \%$ & $74 \%$ & $34 \%$ & $80 \%$ & $16^{\circ} \%$ & $82 \%$ & $15 \%$ \\
\hline \multirow{2}{*}{$\begin{array}{l}\text { Tiempo de } \\
\text { respuesta de } \\
\text { dudas y/o } \\
\text { peticiones }\end{array}$} & ADECUADO & INADECEADO & ADEUCADO & INADECUADO & ADECUADO & INADECEADO & ADECEADO & INADECT \\
\hline & $53 \%$ & $44 \%$ & $60 \%$ & $37 \%$ & $36 \%$ & $40 \%$ & $54 \%$ & $43^{\mathrm{n}}$ \\
\hline \multirow{2}{*}{$\begin{array}{l}\text { Horario de } \\
\text { atención al } \\
\text { alumno }\end{array}$} & SUFICIENTE & INSUFICIENTE & SLFICIENTE & INSUFICIENTE & SUFICIENTE & INSUFICIENTE & SUFICIENTE & INSUFICII \\
\hline & $21^{D_{0}}$ & $78 \%$ & $45 \%$ & $49 \%$ & $38 \%$ & $57^{\circ} \%$ & $41^{10}$ & $56^{\circ} \mathrm{n}$ \\
\hline \multirow[t]{2}{*}{ Trato recibido } & BEENO & ME-IORABLE & BUENO & ME.JORABLE & BUENO & MEIORABLE & BUENO & MEIORA \\
\hline & $33 \%$ & $38 \%$ & $64^{4} i$ & $27 \%$ & $40 \%$ & $43^{\prime \prime}$ & $50 \%$ & $477^{4 \mathrm{n}}$ \\
\hline
\end{tabular}

\section{DISCUSIÓN}

Respecto a la satisfacción de los seminarios prácticos que se realizan en la Universidad, cabe destacar el aumento en un $10 \%$ de la misma en el curso académico 2007/08. Este aumento es debido a la puesta en marcha de una serie de mejoras realizadas como consecuencia de los resultados obtenidos con el análisis de los datos del curso académico 2004/05. Mejoras tales como mayor coordinación entre los profesores que imparten los seminarios prácticos para evitar solapamientos y dinamización de los seminarios. 
Más del $90 \%$ de los alumnos encuestados en ambos cursos académicos no sólo consideran suficientes los materiales utilizados en los seminarios prácticos, sino que también afirman que las técnicas aprendidas en los seminarios prácticos se corresponden con la realidad asistencial y que la realización de los mismos supone una mejora para el inicio de sus prácticas clínicas.

Con respecto a la manipulación del material durante los seminarios por parte del alumno, los datos obtenidos refuerzan la importancia que para el currículum tiene el aprendizaje práctico, que según la Pirámide de Aprendizaje(1) supone una de las mayores tasas de retención. Cabe destacar el aumento de la manipulación de los materiales por parte del alumno en el curso académico 2007/08, motivadas por las mejoras anteriormente citadas.

Cabe destacar la alta satisfacción que manifiestan los alumnos en relación con sus prácticas externas realizadas en centros sanitarios y socio-sanitarios de la Región de Murcia. Parte de este logro se debe a la implicación del profesional - tutor en la formación de pregrado, implicación que se aprecia fácilmente en el análisis de las graficas 5 y 6 donde un porcentaje elevado de alumnos consideran que los tutores están muy capacitados tanto para impartir enseñanza como para evaluar las prácticas, participando activamente y como parte de las actividades de formación, en la integración del alumno en el equipo de trabajo.

Nos ha sorprendido la opinión mayoritaria de los alumnos a favor de que participen en su formación más de un profesional - tutor, en contra de la opinión tanto del claustro de profesores como de los propios tutores de prácticas asistenciales, que piensan que este hecho si bien no supone un perjuicio tampoco un beneficio.

La asignación alumno / profesional - tutor se está llevando a cabo en esta Universidad desde el curso académico 2005/06, decisión tomada a raíz de las conclusiones obtenidas en la Sesión de Trabajo para la Integración Docencia -Asistencia en Enfermería, que se realizó en el curso académico 2004/05.

Tal como explicábamos en la introducción, la gestión de los créditos prácticos se realiza a través de la UPE. Esta gestión es evaluada por los alumnos de manera satisfactoria, ya que consideran adecuados los sistemas de difusión de la información y el tiempo de respuesta de las dudas y peticiones realizadas a la UPE, aunque los datos son poco significativos. Sin embargo, en otros aspectos, tales como el horario de atención al alumno y el trato recibido la mayoría opinan que podría ser mejorable. Creemos que esta opinión se debe principalmente a la concentración de alumnos en el horario de descanso de las clases (11:00 a 11:30h y 18:30 a 19:00h), lo que da lugar a largas colas. Para conseguir una mejora en la atención y trato a los alumnos, en el curso académico 2007/08 se ha fomentado la utilización del correo electrónico y del teléfono para las consultas que no necesitan atención personal.

\section{CONCLUSIONES}

La satisfacción de los alumnos de enfermería de la UCAM con respecto a las prácticas desarrollados durante su formación de pregrado es positiva.

Respecto a las prácticas internas o seminarios prácticos en sala de demostraciones, la satisfacción de los alumnos aumentó en un 10\% del curso académico 2004/05 al curso $2007 / 08$. 
Los alumnos se encuentran altamente satisfechos con las prácticas clínicas que realizan en centros sanitarios y socio-sanitarios de la Región de Murcia durante su formación.

Respecto a las gestiones que se llevan a cabo desde la UPE, los alumnos indican que existen aspectos a mejorar.

Las mejoras establecidas para aumentar la coordinación entre profesores mejoró la satisfacción de los alumnos con respecto a sus prácticas internas.

\section{BIBLIOGRAFÍA}

(1) Bales, E. Pirámide de Aprendizaje. USA: National Training Laboratories; 1996.

(2) Miller G. The assessment of clinical skills/ competence/ performance. Academic Medicine 1990, 65: $63-7$.

(3) Guía docente 2007/2008. Diplomatura Enfermería. Murcia: Universidad Católica San Antonio; 2007.

(4) Guía docente 2004/2005. Diplomatura Enfermería. Murcia: Universidad Católica San Antonio; 2004.

(5) Libro Blanco de la Enfermería Española. Madrid: Consejo General de Enfermería de España; 1999. 